\title{
Poor Sleep Quality Is Associated with Dawn Phenomenon and Impaired Circadian Clock Gene Expression in Subjects with Type 2 Diabetes Mellitus
}

\author{
Yuxin Huang, ${ }^{1,2}$ Haidong Wang, ${ }^{1,2}$ Yuan Li, ${ }^{1,2}$ Xiaoming Tao,, and Jiao Sun $^{1,2}$ \\ ${ }^{1}$ Department of Endocrinology, Huadong Hospital Affiliated to Fudan University, 221 Yananxi Road, Shanghai 200040, China \\ ${ }^{2}$ Shanghai Key Laboratory of Clinical Geriatric Medicine, Shanghai, China
}

Correspondence should be addressed to Jiao Sun; sunjiao6972@aliyun.com

Received 3 November 2016; Revised 16 January 2017; Accepted 30 January 2017; Published 2 March 2017

Academic Editor: Darío Acuña-Castroviejo

Copyright (c) 2017 Yuxin Huang et al. This is an open access article distributed under the Creative Commons Attribution License, which permits unrestricted use, distribution, and reproduction in any medium, provided the original work is properly cited.

\begin{abstract}
Aims. We investigated whether poor sleep quality is associated with both dawn phenomenon and impaired circadian clock gene expression in subjects with diabetes. Methods. 81 subjects with diabetes on continuous glucose monitoring were divided into two groups according to the Pittsburgh Sleep Quality Index. The magnitude of dawn phenomenon was quantified by its increment from nocturnal nadir to prebreakfast. Peripheral leucocytes were sampled from 81 subjects with diabetes and 28 normal controls at 09:00. Transcript levels of circadian clock genes (BMAL1, PER1, PER2, and PER3) were determined by real-time quantitative polymerase chain reaction. Results. The levels of $\mathrm{HbAlc}$ and fasting glucose and the magnitude of dawn phenomenon were significantly higher in the diabetes group with poor sleep quality than that with good sleep quality. Peripheral leucocytes from subjects with poor sleep quality expressed significantly lower transcript levels of BMAL1 and PER1 compared with those with good sleep quality. Poor sleep quality was significantly correlated with magnitude of dawn phenomenon. Multiple linear regression showed that sleep quality and PER1 were significantly independently correlated with dawn phenomenon. Conclusions. Dawn phenomenon is associated with sleep quality. Furthermore, mRNA expression of circadian clock genes is dampened in peripheral leucocytes of subjects with poor sleep quality.
\end{abstract}

\section{Introduction}

The term "dawn phenomenon" was first introduced by Schmidt et al. in 1981 and was used to describe fasting hyperglycemia or a spontaneous rise in insulin requirements during the early morning, occurring in the absence of nocturnal hypoglycemia [1]. Dawn phenomenon mostly affects children and youth with type 1 diabetes, although it is not rare in subjects with type 2 diabetes (as high as $40 \%$, reported by Monnier et al.) [2]. By using a continuous glucose monitoring system, dawn phenomenon could be determined by the difference between nocturnal nadir and prebreakfast glucose levels [2]. Monnier et al. published that the approximate impact of the dawn phenomenon on $\mathrm{HbAlc}$ level was $0.4 \%$ and the impact for averaged $24 \mathrm{~h}$ mean glucose concentrations was $12.4 \mathrm{mg} / \mathrm{dL}$ [3]. Furthermore, fasting glucose levels are one of the most important targets in the management of type 2 diabetes, and these can be affected by the dawn phenomenon. Thus, we should not ignore this problem.

The mechanism of dawn phenomenon still remains unclear. It is generally believed that dawn phenomenon is a result of pancreatic beta-cell dysfunction, which increases endogenous glucose production, persistent insulin resistance, and hepatic glucose output. Some endogenous hormones, especially growth hormone, cortisol, and glucagon, also take part in the pathophysiological mechanism of dawn phenomenon $[4,5]$. In addition, age was reported to have an independent effect on the dawn phenomenon [2]. It is well known that poor sleep quality is common in elderly subjects. Sleep disorder is associated with insulin resistance and impaired glucose tolerance in multiple metabolic pathways in healthy subjects or in subjects with diabetes [6]. Furthermore, obstructive sleep apnea-hypopnea syndrome 
(OSAHS) is also associated with insulin resistance, and it is common in subjects with diabetes. Thus, we believe poor sleep quality is one of the core mechanisms in dawn phenomenon.

On the other hand, mechanisms that impair the circadian clock genes, such as circadian locomotor output cycles kaput (CLOCK), brain and muscle Arnt-like protein 1 (BMAL1), and period genes (PER1, PER2, and PER3), contribute to defective beta-cell function and development of type 2 diabetes [7-12]. In mammals, the circadian system exists in almost all tissues, including hypothalamic suprachiasmatic nucleus (SCN, regarded as the central clock), liver, pancreas, kidney, and leucocytes [13]. Circadian clock has a vital impact on the regulation of physiological and biochemical processes, cellular and energy metabolism, glucose and lipid homeostasis, and feeding behavior. Disruption of these clock genes affects locomotor activity, feeding behavior, metabolism, and glucose homeostasis [14]. Therefore, we speculate that disruption of circadian clock genes may also indirectly increase glucose levels during the early morning.

Publications have shown that circadian clock genes are associated with sleep outcomes in human research $[15,16]$. Therefore, it is interesting to find an interaction between dawn phenomenon, sleep quality, and circadian clock gene expression in subjects with diabetes. To the best of our knowledge, there is a lack of robust evidence to support this relationship. Thus, we felt it was imperative to assess this issue. In the present study, we investigated the following: (a) whether sleep quality is associated with dawn phenomenon and (b) whether dawn phenomenon is correlated with impaired circadian clock gene expression in subjects with type 2 diabetes. These results may help us to recognize a new pathophysiological mechanism of dawn phenomenon and find a new target for management and treatment of type 2 diabetes.

\section{Materials and Methods}

All participants were recruited from Department of Endocrinology, Huadong Hospital Affiliated to Fudan University, Shanghai, People's Republic of China, from March 2014 to July 2015. There were, in total, 81 subjects with type 2 diabetes with poor sleep quality or with good sleep quality and 28 normal controls with good sleep quality enrolled in the study according to the protocol. The study protocol was approved by the Ethics Committee of Huadong Hospital. All procedures performed in studies involving human participants were in accordance with the ethical standards of the institutional research committee and with the 1964 Helsinki declaration. Informed consent was obtained from all individual participants included in the study.

\subsection{Inclusion Criteria. The inclusion criteria were comprised} of the following.

(1) All participants with diabetes were diagnosed with type 2 diabetes according to the 2014 American Diabetes Association criteria [17]. (2) All subjects with diabetes received stable treatment with dietary specifications alone or with oral hypoglycemic agents (OHAs, including metformin, $\alpha$-glucosidase inhibitors, sulfonylureas, glinides, and dipeptidyl peptidase- 4 inhibitors or in a combination of 2 OHAs) for at least 3 months.

Normal controls were determined to be healthy participants without diabetes, impaired glucose tolerance, poor sleep quality, or a history of OSAHS.

2.2. Exclusion Criteria. The exclusion criteria included the following.

(1) Subjects received insulin therapy in 3 months prior to the study; (2) they had an HbAlc level of more than $75 \mathrm{mmol} / \mathrm{mol}$ (9.0\%); (3) they were currently afflicted with diabetic ketoacidosis or hyperosmolar coma; (4) they were currently afflicted with cardiovascular disease or other serious diseases; (5) they were currently afflicted with hypoglycemia or suspected hypoglycemia; (6) they had a creatinine clearance rate of $<45 \mathrm{~mL} / \mathrm{min}$; (7) they had impaired liver function (liver enzymes more than twice the upper limit of normal); (8) they were currently receiving any hypnagogue; (9) they should be in poor compliance with diabetes treatment; or (10) they had a history of OSAHS.

\subsection{Clinical Investigations and Laboratory Determinations.} All participants were instructed to maintain their recommended dietary, medication, and exercise programs. Following a $10 \mathrm{~h}$ overnight fast, anthropometric, blood pressure, serum, and plasma samples were collected. Biochemical measurements of plasma glucose, insulin, HbA1c, serum lipids, and plasma cortisol were performed in a central laboratory. Insulin sensitivity was calculated as the homeostasis model assessment of insulin resistance index (HOMA-IR) by using the HOMA Calculator (Headington, Oxford, UK) (http://www.dtu.ox.ac.uk).

2.4. Sleep Quality. A total of 81 subjects with type 2 diabetes were divided into two groups according to the Pittsburgh Sleep Quality Index (PSQI), including a diabetes group with poor sleep quality and a diabetes group with good sleep quality [18]. PSQI is a self-reported questionnaire that assesses sleep quality and disorders. It contains seven component scores: subjective sleep quality, sleep latency, sleep duration, habitual sleep efficiency, sleep disturbances, use of sleeping medication, and daytime dysfunction. The sum of the scores for these seven components determined sleep quality classification: more than 7 points indicated poor sleep quality and less than or equal to 7 points indicated good sleep quality (the threshold of poor sleep quality was 7 points in Chinese people as reported [19]). All participants had 15 min to complete the questionnaire under the guidance of one trained researcher.

2.5. Continuous Glucose Monitoring. All 81 subjects with diabetes were evaluated using a CGMS for 72 hours (MiniMed system, Medtronic Inc., USA). Participants were obliged to input their capillary blood glucose four times a day for adjusting the CGMS. Three meals were required to be eaten between 7:00 and 7:30,11:00 and 11:30, and 17:00 and 17:30, respectively. The sensor of CGMS was installed on day 0 and removed on day 3 . The data provided from CGMS were obtained during day 1 and day 2 to avoid any 
interference due to installation and removal of the sensor. Furthermore, the glucose values recorded on day 1 and day 2 were averaged in order to avoid bias. The nocturnal nadir and prebreakfast glucose values were quantified by the averaged CGMS data from day 1 to day 2. The magnitude of dawn phenomenon ( $\triangle$ Dawn) was quantified by its increment from nocturnal nadir to prebreakfast glucose level, and the threshold of $\Delta$ Dawn was determined to be $20 \mathrm{mg} / \mathrm{dL}$ $(1.11 \mathrm{mmol} / \mathrm{L})$ according to Monnier et al. [3]. If all glucose measurements at night were higher than the prebreakfast measurement, $\Delta$ Dawn level was considered to be 0 .

2.6. RNA Preparation and Real-Time Quantitative PCR. Blood samples were taken from the forearm vein at 09:00 [7]. Leucocytes were isolated immediately using lymphocyte separation media (Dakewe Biotech, Shenzhen, China). The isolation of total RNA was performed using the RNAiso Plus reagent (TaKaRa, Otsu, Japan) according to the manufacturer's instructions. $1 \mu \mathrm{g}$ of total RNA was treated with RNase-free DNase I for 10 minutes at $37^{\circ} \mathrm{C}$ to remove any contaminating DNA and reverse-transcribed with AMV reverse transcriptase (TaKaRa, Otsu, Japan). Gene expression was analyzed by real-time quantitative PCR using the ABI PRISM 7000 Sequence Detection System (Applied Biosystems). The GenBank accession numbers, assay ID, and the target exons were as follows: NM_001178.4 and Hs00154147_m1, 9-10 for BMAL1; NM_002616.1 and Hs00242988_m1, 22-23 for PER1; NM_022817.1 and Hs00256143_m1, 8-9 for PER2; and NM_016831.1 and Hs00213466_m1, 15-16 for PER3. Relative mRNA levels of each transcript were determined by the comparative $\mathrm{Ct}$ method with the reference gene (GAPDH).

2.7. Statistical Analysis. Continuous variables were expressed as the mean $\pm \mathrm{SD}$ and were analyzed using ANOVA and post hoc analysis for normally distributed data. HbAlc measurements were reported in International Federation of Clinical Chemistry and Laboratory Medicine (IFCC) units ( $\mathrm{mmol} / \mathrm{mol}$ ) followed by derived National Glycohemoglobin Standardization Program (NGSP) units (\%). Categorical variables were expressed as numbers (\%) and analyzed by using the chi-squared test and Fisher's exact test. Differences in clock gene expression were analyzed between the diabetes group with poor sleep quality, diabetes group with good sleep quality, and normal controls using the Mann-Whitney test. Correlations were calculated using Spearman's correlation coefficient. All $P$ values were 2 -tailed and $P$ values less than 0.05 were considered significant. The enter method of multiple linear regression analysis was used to determine the association of $\triangle$ Dawn level with age, BMI, PQSI, and expression levels of PER1, PER2, PER3, and BMAL1. Variables were entered into the model if the significance of the $F$ value was less than 0.05 and were removed if it was more than 0.1. Statistical analyses were performed with SPSS version 13.0 (SPSS Inc., Chicago, IL, USA). All enrolled subjects were identified by participant numbers in the database to ensure anonymity. All artworks were created by GraphPad Prism 5 (GraphPad Software Inc., San Diego, CA).

\section{Results and Discussion}

3.1. Clinical Characteristics. There were, in total, 81 subjects with diabetes and 28 normal controls (15 males and 13 females) enrolled in the study. All participants had completed the whole study. The clinical characteristics of the 109 participants are summarized in Table 1. According to the Pittsburgh Sleep Quality Index, 81 subjects with diabetes had been separated into two groups: 37 with poor sleep quality (PSQI more than 7 points, 21 males and 16 females) and 44 with good sleep quality (PSQI less than or equal to 7 points, 24 males and 20 females). Gender, age, body mass index, blood pressure, fasting insulin, postbreakfast insulin, HOMA-IR, lipid profile, and plasma cortisol level showed no significant differences between the two groups. When compared with normal controls, the diabetes group with poor sleep quality showed a significantly higher level of systolic blood pressure, diastolic blood pressure, fasting glucose, HbA1c, and HOMA-IR (all $P<0.01$ ).

3.2. Analysis of $24 \mathrm{~h}$ Glycemic Profile and Dawn Phenomenon. All subjects with diabetes were evaluated over 3 days using a CGMS. The $24 \mathrm{~h}$ glycemic profile, nocturnal nadir glucose, and magnitude of the dawn phenomenon $(\Delta \mathrm{Dawn})$ are summarized in Table 1. The diabetes group with poor sleep quality presented with higher fasting glucose, postbreakfast glucose, $24 \mathrm{~h}$ mean glucose level, and HbAlc level than that with good sleep quality (all $P<0.05$ ). The glucose levels at prelunch, postlunch, predinner, postdinner, and nocturnal nadir showed no statistical difference between the two diabetes groups. Following the determination of a $\Delta$ Dawn threshold as $20 \mathrm{mg} / \mathrm{dL}, 37$ of 81 subjects with diabetes were categorized as sufferers from dawn phenomenon; thus, the prevalence of dawn phenomenon in our study is $45.7 \%$. The diabetes group with poor sleep quality had a higher $\Delta$ Dawn level $(P=0.001)$ and higher proportion $(P<0.001)$ of dawn phenomenon than that with good sleep quality (see Figure 1 and Table 1).

3.3. Gene Expression Assays. The transcript levels of these four clock genes (BMAL1, PER1, PER2, and PER3) in subjects with diabetes and normal controls were compared, as shown in Figure 2. The transcript levels of BMAL1, PER1, and PER3 were significantly lower (all $P<0.01$ ) in the diabetes group with poor sleep quality compared to the normal control group. Interestingly, the mRNA levels of BMAL1 and PER1 genes were found to be significantly lower (both $P<0.01$ ) in the diabetes group with poor sleep quality compared to that with good sleep quality. In contrast, the transcript levels of the PER2 and PER3 had not display any significant difference between the two diabetes groups.

3.4. Correlation and Regression Analysis. The correlation analysis showed that PQSI is significantly correlated with $\Delta$ Dawn level $(r=0.494, P<0.001)$. Among the four circadian clock genes tested in our study, the expression of PER1 and BMAL1 was significantly negatively correlated with PQSI $(r=-0.3, P=0.002 ; r=-0.31, P=0.001)$, as noted in Figures 3(a) and 3(b). Furthermore, the expression 
TABLE 1: Characteristics and glucose profiles of the study population, normal controls, and the diabetes group with poor sleep quality and with good sleep quality.

\begin{tabular}{|c|c|c|c|c|c|}
\hline Variables & DM group with poor sleep quality & DM group with good sleep quality & ${ }^{\mathrm{a}} P$ value & Control group & ${ }^{a} P$ value \\
\hline No. (M/F) & $37(21 / 16)$ & $44(24 / 20)$ & 0.565 & $28(15 / 13)$ & 0.421 \\
\hline Age (years) & $66.2 \pm 8.8$ & $65.6 \pm 10.3$ & 0.774 & $64.43 \pm 6.7$ & 0.364 \\
\hline BMI $\left(\mathrm{kg} / \mathrm{m}^{2}\right)$ & $24.8 \pm 4.1$ & $24.4 \pm 3.0$ & 0.588 & $23.2 \pm 2.7$ & 0.054 \\
\hline PSQI & $10.1 \pm 1.7$ & $4.1 \pm 1.7$ & $<0.001$ & $4.0 \pm 1.5$ & $<0.001$ \\
\hline Systolic BP (mmHg) & $132.0 \pm 13.1$ & $129.9 \pm 10.9$ & 0.452 & $121.7 \pm 13.7$ & 0.003 \\
\hline Diastolic BP (mmHg) & $78.7 \pm 7.8$ & $77.6 \pm 7.0$ & 0.498 & $73.0 \pm 7.9$ & 0.006 \\
\hline $\mathrm{HbA1c}(\mathrm{mmol} / \mathrm{mol})$ & $56 \pm 5$ & $53 \pm 7$ & 0.032 & $37 \pm 4$ & $<0.001$ \\
\hline$(\%)$ & $7.3 \pm 0.5$ & $7.0 \pm 0.6$ & & $5.5 \pm 0.4$ & \\
\hline Fasting insulin $(\mu \mathrm{U} / \mathrm{mL})$ & $7.9 \pm 4.5$ & $8.3 \pm 4.4$ & 0.716 & $6.7 \pm 3.0$ & 0.189 \\
\hline Postbreakfast insulin $(\mu \mathrm{U} / \mathrm{mL})$ & $27.7 \pm 16.1$ & $25.9 \pm 14.1$ & 0.513 & $23.6 \pm 14.2$ & 0.325 \\
\hline HOMA-IR & $2.6 \pm 1.4$ & $2.4 \pm 1.5$ & 0.674 & $1.5 \pm 0.7$ & $<0.001$ \\
\hline Triglyceride (mmol/L) & $1.8 \pm 1.5$ & $1.5 \pm 1.3$ & 0.357 & $1.5 \pm 0.9$ & 0.233 \\
\hline Total cholesterol (mmol/L) & $4.6 \pm 1.1$ & $4.7 \pm 1.2$ & 0.682 & $5.0 \pm 1.2$ & 0.121 \\
\hline $\mathrm{HDL}-\mathrm{C}(\mathrm{mmol} / \mathrm{L})$ & $1.3 \pm 0.3$ & $1.4 \pm 0.3$ & 0.301 & $1.3 \pm 0.4$ & 0.812 \\
\hline LDL-C (mmol/L) & $2.4 \pm 0.9$ & $2.4 \pm 0.9$ & 0.933 & $2.8 \pm 0.9$ & 0.084 \\
\hline Plasma cortisol (nmol/L) & $419.3 \pm 89.5$ & $384.1 \pm 112.0$ & 0.155 & $408.9 \pm 85.3$ & 0.673 \\
\hline Fasting glucose (mg/dL) & $133.2 \pm 21.6$ & $120.6 \pm 23.4$ & 0.001 & $86.4 \pm 10.8$ & $<0.001$ \\
\hline Postbreakfast (mg/dL) & $217.2 \pm 35.8$ & $183.6 \pm 22.1$ & 0.032 & N/A & N/A \\
\hline Prelunch (mg/dL) & $135.7 \pm 32.6$ & $127.9 \pm 30.6$ & 0.380 & N/A & N/A \\
\hline Postlunch (mg/dL) & $162.3 \pm 30.2$ & $147.2 \pm 28.44$ & 0.056 & N/A & N/A \\
\hline Predinner (mg/dL) & $134.3 \pm 33.4$ & $131.4 \pm 33.5$ & 0.814 & N/A & N/A \\
\hline Postdinner (mg/dL) & $166.6 \pm 28.2$ & $155.7 \pm 24.5$ & 0.444 & N/A & N/A \\
\hline Nadir glucose (mg/dL) & $106.5 \pm 17.8$ & $106.2 \pm 18.2$ & 0.642 & N/A & N/A \\
\hline $24 \mathrm{~h}$ mean glucose $(\mathrm{mg} / \mathrm{dL})$ & $175.2 \pm 30.6$ & $148.5 \pm 20.7$ & 0.028 & N/A & N/A \\
\hline$\Delta$ Dawn $(\mathrm{mg} / \mathrm{dL})$ & $26.5 \pm 13.1$ & $14.4 \pm 12.8$ & 0.001 & N/A & N/A \\
\hline Dawn phenomenon (\%) & $29(78.4)$ & $8(18.2)$ & $<0.001$ & N/A & N/A \\
\hline
\end{tabular}

Data are means \pm SD or number (percentage).

${ }^{\mathrm{a}} P$ value compared to the DM group with poor sleep quality.

DM: diabetes mellitus; BMI: body mass index; BP: blood pressure; PSQI: Pittsburgh Sleep Quality Index; HDL-C: high-density lipoprotein cholesterol; LDL-C: low-density lipoprotein cholesterol; HOMA-IR: homeostasis model assessment of insulin resistance; N/A: not available; $\triangle$ Dawn: difference between prebreakfast and nocturnal nadir glucose values; Dawn phenomenon: $\Delta$ Dawn more than $20 \mathrm{mg} / \mathrm{dL}$.

of PER1 and BMAL1 was significantly negatively correlated with $\triangle$ Dawn level $(r=-0.29, P=0.008 ; r=-0.45$, $P=0.001$ ), as noted in Figures $3(\mathrm{c})$ and $3(\mathrm{~d})$. Unfortunately, the level of HOMA-IR showed no correlation with either PQSI $(r=0.096, P=0.319)$ or $\triangle$ Dawn level ( $r=-0.095, P=0.401)$.

The multiple linear regression analysis enter method was used to determine the association of $\triangle D$ awn with age, BMI, PQSI, and gene expression levels of PER1, PER2, $P E R 3$, and BMAL1. Multiple variable linear regression showed that PQSI and expression level of PER1 were significantly independently correlated with $\triangle$ Dawn level (adjusted $\left.R^{2}=0.325, P=0.001\right)$. The standard $\beta$-coefficients from the regression model of PQSI and PER1 were 0.112 and -0.086 , respectively.

\section{Discussion}

The term "dawn phenomenon" has been in use for more than 30 years, but endocrinologists seem to be uninterested in this area of study. With the development and wide use of CGMS, more and more scholars begin to pay attention to this phenomenon. Monnier et al. studied dawn phenomenon in three groups of subjects with type 2 diabetes (on diets alone, on insulin sensitizers alone, and on insulin secretagogues alone or in combination with insulin sensitizers) by using CGMS. The approximate impact of dawn phenomenon on HbAlc level was $0.4 \%$, and the impact on the averaged $24 \mathrm{~h}$ mean glucose concentrations was $12.4 \mathrm{mg} / \mathrm{dL}$ [3]. Interestingly, their study showed a similar $\Delta$ Dawn level in all three groups. These results revealed that dawn phenomenon was important in the management of type 2 diabetes and that it could not be eliminated by any of the current oral antidiabetes agents. This means that dawn phenomenon could only be controlled by using insulin, especially by using basal insulin such as neutral protamine Hagedorn (NPH), long-acting insulin analogs (LA-IA), or continuous subcutaneous insulin infusion (CSII) [20]. However, hypoglycemia occurred frequently in this population using CSII or using $\mathrm{NPH}$ as reported. This seems to be an inevitable side 


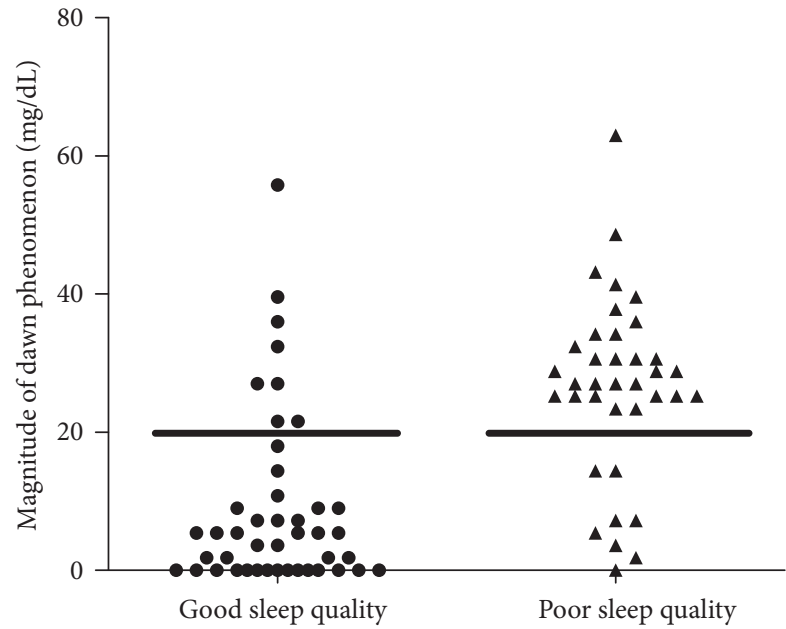

Figure 1: Magnitude of dawn phenomenon in subjects with diabetes. Black circle: diabetes group with good sleep quality; black triangle: diabetes group with poor sleep quality. When the threshold was settled at $20 \mathrm{mg} / \mathrm{dL}$, the prevalence of dawn phenomenon in the two groups was $18.2 \%$ versus $78.4 \%(P<0.001)$.

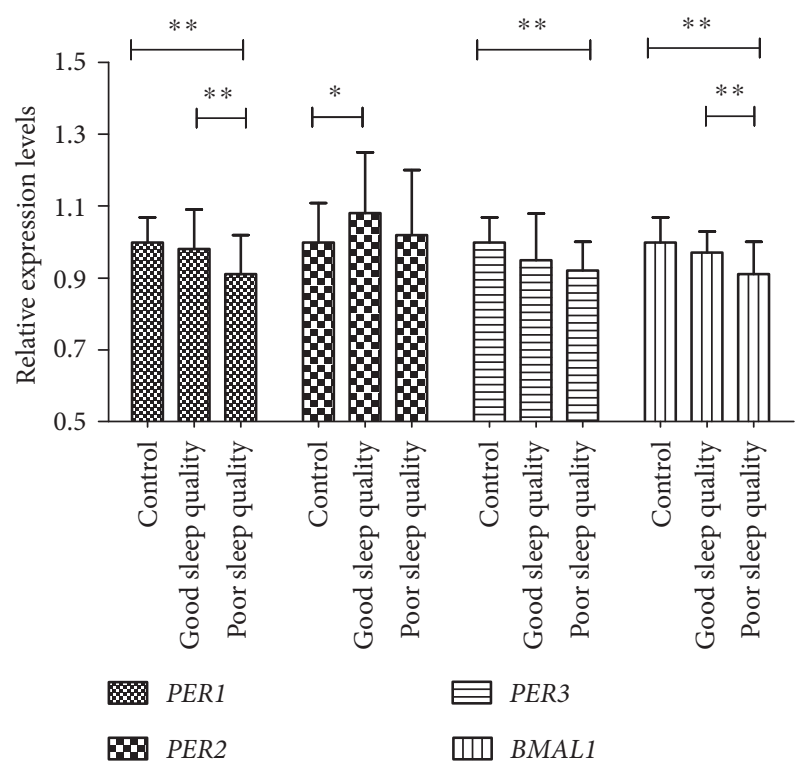

FIGURE 2: Transcript levels of the circadian clock genes in peripheral leucocytes of subjects with diabetes and normal controls. Peripheral leucocytes were obtained from 28 healthy controls, 37 diabetes subjects with poor sleep quality, and 44 diabetes subjects with good sleep quality at 09:00 hours. Transcript levels of the circadian clock genes were determined by real-time quantitative PCR. The mean value of the healthy control was set to 1 for each gene. ${ }^{*} P<0.05,{ }^{* *} P<0.01$. The errors bars represented SD.

effect of using insulin in the treatment of dawn phenomenon [21, 22]. Thus, it is important for us to determine the mechanism leading to dawn phenomenon and to find a new way to manage fasting hyperglycemia.

In our study, 37 of 81 subjects with type 2 diabetes suffered from dawn phenomenon, and the prevalence of dawn phenomenon was $45.7 \%$. After grouping the subjects by a PQSI of more than 7 points, the mean $\triangle$ Dawn in the diabetes group with poor sleep quality was $26.5 \pm 13.1 \mathrm{mg} / \mathrm{dL}$, significantly higher than that in the diabetes group with good sleep quality $(14.4 \pm 12.8 \mathrm{mg} / \mathrm{dL}, P=0.001)$. The diabetes group with poor sleep quality had a dramatically higher proportion of subjects with dawn phenomenon than that with good sleep quality $(78.4 \%$ versus $18.2 \%, P<0.001)$. Furthermore, correlation analysis indicated a significant relationship between sleep quality and $\Delta$ Dawn level. Multiple variable linear regression also showed that sleep quality was significantly independently correlated with $\Delta$ Dawn level after adjusting for age and BMI. It was very interesting to find that subjects with poor sleep quality presented with a higher fasting glucose, postbreakfast glucose, $24 \mathrm{~h}$ mean glucose level, and HbA1c levels than those with good sleep quality, while the glucose level at prelunch, postlunch, predinner, postdinner, and nocturnal nadir showed no significant difference between the two groups in our study. We could speculate that poor sleep quality has a greater influence on fasting and postbreakfast glucose than on other glucose levels.

It has been well published that sleep disturbances, sleep insufficiency, or sleep fragmentation has a relationship with abnormal glucose metabolism and increased diabetes risk. Prospective studies had revealed that sleep disorders were associated with an increased risk of incident diabetes [23]. However, few studies have presented a link between poor sleep quality and dawn phenomenon. Monnier et al. studied eighty-one individuals with type 2 diabetes and divided them into three subgroups by age (group $1: \geq 70$ years, group 2: 60-69 years, and group 3: $\leq 59$ years). HbAlc levels showed no statistical significance across the three groups, while $\Delta$ Dawn levels were different in the three groups: $22.0 \pm 4.7 \mathrm{mg} / \mathrm{dL}$ in group $1,21.3 \pm 3.6 \mathrm{mg} / \mathrm{dL}$ in group 2 , and $18.0 \pm 3.6 \mathrm{mg} / \mathrm{dL}$ in group 3 [2]. The results argued that dawn phenomenon was present in the elderly but that this population might be frequently suffered from sleep disturbance. A study in China showed that dawn phenomenon was closely associated with obesity and insulin resistance, and the frequency of dawn phenomenon increased with body mass index [24]. It has been well known that obesity is correlated with OSAHS, which combines hypoxemia and sleep fragmentation and is a major risk factor for insulin resistance and possibly diabetes [23]. Furthermore, a human study showed that exogenous growth hormone injection in the early evening decreased the metabolic clearance rate of insulin and reduced insulin sensitivity in subjects with type 2 diabetes. The results provided direct evidence for the role of the growth hormone, which is secreted mostly during sleep, in regulation of insulin sensitivity and in causation of dawn phenomenon in subjects with type 2 diabetes [5]. Another study in China provided direct evidence of the association between poor sleep quality and dawn phenomenon. Ren et al. [19] demonstrated that the levels of HbAlc, fructosamine, increment of fasting glucose and nocturnal nadir glucose, $24 \mathrm{~h}$ mean glucose, fasting insulin, HOMA-IR, and area under curve of insulin were significantly higher in the diabetes subjects with poor 


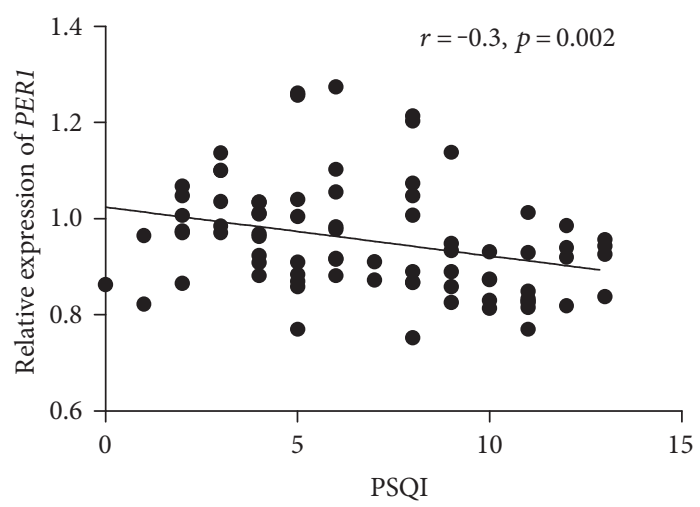

(a)

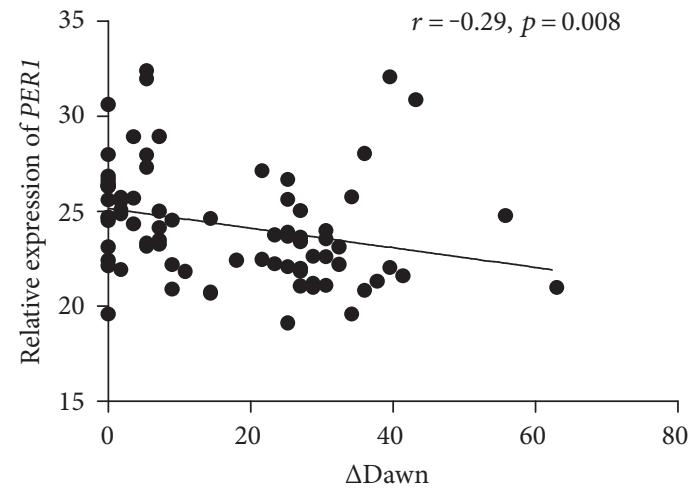

(c)

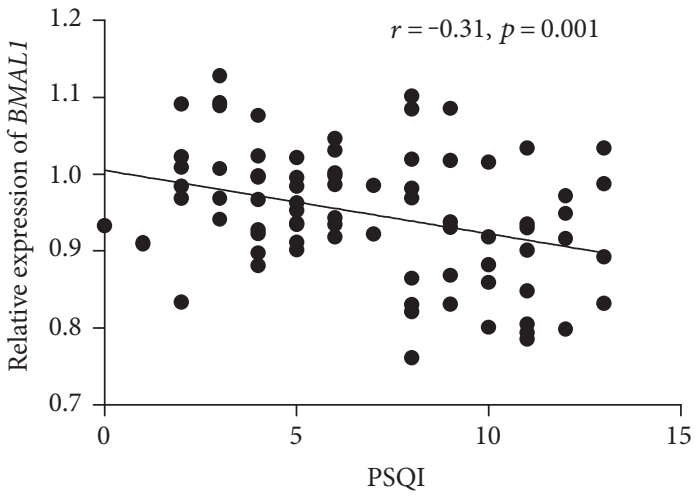

(b)

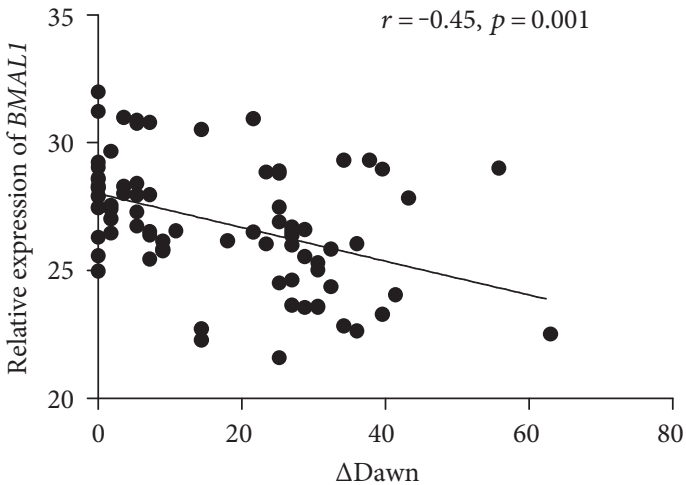

(d)

FIgURe 3: Relationships between Pittsburgh Sleep Quality Index and mRNA levels of PER1 (a) and BMAL1 (b). Relationships between $\triangle$ Dawn and mRNA levels of PER1 (c) and BMAL1 (d).

sleep quality than in those with good sleep quality. In addition, poor sleep quality was positively correlated with the glucagon-to-insulin ratio. These results suggested that poor sleep quality could be one of the main causes of dawn phenomenon and that dysfunction of both islets $\alpha$-cell and $\beta$-cell was involved in this pathophysiology.

Circadian disruption has been considered to be associated with $\beta$-cell dysfunction, obesity, and type 2 diabetes in both animal studies and human studies [25]. Shift work, which has been regarded as a common cause of circadian disruption in modern society, has been proven to be correlated with an increased risk of type 2 diabetes by prospective cohort studies [26-28]. Circadian clock genes are important in the regulation of glucose homeostasis. The CLOCK and $B M A L 1$ complex is the transcription factor that acts as a positive regulator of circadian clock gene expression, such as regulation of the period and cryptochrome families, while period and cryptochrome proteins feedback and inhibit their own expression and other clock genes [29] (see Figure 4). Impairment of the circadian clock genes such as CLOCK, $B M A L 1$, cryptochrome (CRY1 and CRY2), and period (PER1, PER2, and PER3) contributes to the decrease in glucose tolerance, defective $\beta$-cell function, and the development of type 2 diabetes. Whole-body BMAL1 knockout mice presented with diminished gluconeogenesis and could not recover from insulin-induced hypoglycemia [30]. Mice

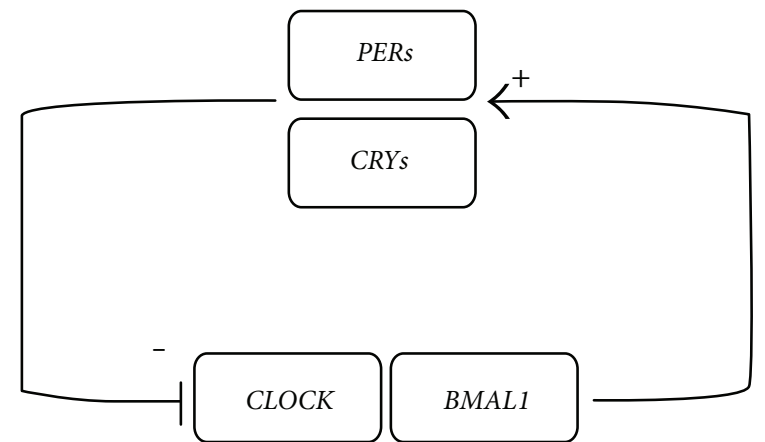

FIgURE 4: The core mechanism of a circadian clock gene. The CLOCK-BMAL1 heterodimer is the core of circadian clock. It stimulates the transcription of period (PERs, including PER1, $P E R 2$, and PER3) and cryptochrome (CRYs, including CRY1 and $C R Y 2)$. Soon afterwards, PERs and CRYs translocate to the nucleus and inhibit CLOCK-BMAL1 activity.

deficient of PER2 had markedly increased circulatory levels of insulin and decreased insulin clearance compared with wild-type mice. Insulin secretion was more effectively stimulated by glucose in PER2-deficient mice [31]. Genetic loss of CRY1/CRY2 results in decreased glucose intolerance, increased glucose level, and constitutively high levels of 
circulating corticosterone in mice [32]. In a human study, leucocytes sampled from subjects with type 2 diabetes expressed significantly lower transcript levels of $B M A L 1$, PER1, and PER3 compared with leucocytes from normal controls; moreover, transcript expression was inversely correlated with HbA1c levels [7].

In our study, the transcript levels of BMAL1, PER1, and PER3 were also significantly lower in the diabetes groups compared to the normal control group. More importantly, the mRNA levels of BMAL1 and PER1 genes were found to be significantly lower in the diabetes group with poor sleep quality compared to that with good sleep quality. In addition, the expression of PER 1 and BMAL1 was negatively correlated with $\triangle$ Dawn level. To the best of our knowledge, this is the first study to discuss the relationship between dawn phenomenon and circadian clock genes. Poor sleep quality and the impairment of circadian clock seem to be closely involved in the pathophysiology of dawn phenomenon in type 2 diabetes. Because poor sleep quality is mostly a controllable ailment, it could be a new target for the management and treatment of dawn phenomenon and type 2 diabetes.

However, our study still has limitations that should be mentioned. The most important limitation is that it was a cross-sectional trial. It remains difficult to discern the "eggs and chickens" among the parameters that were tested. And we could not evaluate the long-term influence of poor sleep quality on dawn phenomenon. In addition, polysomnography has not been used in our study; thus, we could not exclude the influence of OSAHS on our results. Further interventional trials need to be designed. It would be interesting to demonstrate whether changes in sleep quality are or are not associated with changes in the magnitude of the dawn phenomenon and expression levels of circadian clock genes.

\section{Conclusions}

These results of our study showed that poor sleep quality is associated with dawn phenomenon in subjects with type 2 diabetes. Furthermore, mRNA expression of circadian clock genes such as PER1 and BMAL1 is dampened in peripheral leucocytes of diabetes subjects with poor sleep quality. Sleep quality and expression level of PER1 are independent factors of dawn phenomenon. These results argued that cross talk between sleep quality, dawn phenomenon, and circadian clock gene may be an important pathophysiological mechanism in type 2 diabetes. Even though this study is observational in design, such results seem to indicate that poor sleep quality in type 2 diabetes could be responsible for both an overexpression of the dawn phenomenon and an underexpression of circadian clock genes.

\section{Conflicts of Interest}

The authors declare that there is no conflict of interest regarding the publication of this paper.

\section{Authors' Contributions}

Yuxin Huang and Haidong Wang contributed equally to this work.

\section{Acknowledgments}

The authors thank $\mathrm{Wu}$ Wen for questionnaire data collection. This article is supported by Shanghai Municipal Commission of Health and Family Planning, Key Developing Disciplines (2015ZB0501).

\section{References}

[1] M. I. Schmidt, A. Hadji-Georgopoulos, M. Rendell, S. Margolis, and A. Kowarski, "The dawn phenomenon, an early morning glucose rise: implications for diabetic intraday blood glucose variation," Diabetes Care, vol. 4, no. 6, pp. 579-585, 1981.

[2] L. Monnier, C. Colette, M. Sardinoux, G. Baptista, A. Regnier-Zerbib, and D. Owens, "Frequency and severity of the dawn phenomenon in type 2 diabetes: relationship to age," Diabetes Care, vol. 35, no. 12, pp. 2597-2599, 2012.

[3] L. Monnier, C. Colette, S. Dejager, and D. Owens, "Magnitude of the dawn phenomenon and its impact on the overall glucose exposure in type 2 diabetes: is this of concern?" Diabetes Care, vol. 36, no. 12, pp. 4057-4062, 2013.

[4] M. F. Carroll, K. J. Hardy, M. R. Burge, and D. S. Schade, "Frequency of the dawn phenomenon in type 2 diabetes: implications for diabetes therapy," Diabetes Technology \& Therapeutics, vol. 4, no. 5, pp. 595-605, 2002.

[5] K. C. Shih, S. H. Hsieh, C. F. Kwok, C. M. Hwu, P. S. Hsieh, and L. T. Ho, "Effect of growth hormone on dawn phenomenon in patients with type 2 diabetes," Growth Factors, vol. 31, no. 2, pp. 66-73, 2013.

[6] E. Donga, M. van Dijk, J. G. van Dijk et al., "A single night of partial sleep deprivation induces insulin resistance in multiple metabolic pathways in healthy subjects," The Journal of Clinical Endocrinology and Metabolism, vol. 95, no. 6, pp. 2963-2968, 2010.

[7] H. Ando, T. Takamura, N. Matsuzawa-Nagata et al., "Clock gene expression in peripheral leucocytes of patients with type 2 diabetes," Diabetologia, vol. 52, no. 2, pp. 329-335, 2009.

[8] B. Marcheva, K. M. Ramsey, E. D. Buhr et al., "Disruption of the clock components CLOCK and BMAL1 leads to hypoinsulinaemia and diabetes," Nature, vol. 466, no. 7306, pp. $627-631,2010$.

[9] J. Lee, M. Moulik, Z. Fang et al., "Bmall and beta-cell clock are required for adaptation to circadian disruption, and their loss of function leads to oxidative stress-induced beta-cell failure in mice," Molecular and Cellular Biology, vol. 33, no. 11, pp. 2327-2338, 2013.

[10] K. Rakshit, T. W. Hsu, and A. V. Matveyenko, "Bmall is required for beta cell compensatory expansion, survival and metabolic adaptation to diet-induced obesity in mice," Diabetologia, vol. 59, no. 4, pp. 734-743, 2016.

[11] M. Perelis, K. M. Ramsey, B. Marcheva, and J. Bass, "Circadian transcription from beta cell function to diabetes pathophysiology," Journal of Biological Rhythms, vol. 31, no. 4, pp. 323-336, 2016.

[12] K. Rakshit, A. P. Thomas, and A. V. Matveyenko, "Does disruption of circadian rhythms contribute to beta-cell failure 
in type 2 diabetes?" Current Diabetes Reports, vol. 14, no. 4, p. 474, 2014.

[13] K. Rakshit, J. Qian, C. S. Colwell, and A. V. Matveyenko, “The islet circadian clock: entrainment mechanisms, function and role in glucose homeostasis," Diabetes, Obesity \& Metabolism, vol. 17, Supplement 1, pp. 115-122, 2015.

[14] H. Ikeda, Q. Yong, T. Kurose et al., "Clock gene defect disrupts light-dependency of autonomic nerve activity," Biochemical and Biophysical Research Communications, vol. 364, no. 3, pp. 457-463, 2007.

[15] K. A. Lee, C. Gay, E. Byun, A. Lerdal, C. R. Pullinger, and B. E. Aouizerat, "Circadian regulation gene polymorphisms are associated with sleep disruption and duration, and circadian phase and rhythm in adults with HIV," Chronobiology International, vol. 32, no. 9, pp. 1278-1293, 2015.

[16] T. D. Viena, C. M. Gobin, A. I. Fins, T. J. Craddock, A. Tartar, and J. L. Tartar, "A PER3 polymorphism interacts with sleep duration to influence transient mood states in women," Journal of Circadian Rhythms, vol. 14, no. 1, p. 3, 2016.

[17] American Diabetes Association, "Diagnosis and classification of diabetes mellitus," Diabetes Care, vol. 37, Supplement 1, pp. S81-S90, 2014.

[18] D. J. Buysse, C. F. Reynolds 3rd, T. H. Monk, S. R. Berman, and D. J. Kupfer, "The Pittsburgh Sleep Quality Index: a new instrument for psychiatric practice and research," Psychiatry Research, vol. 28, no. 2, pp. 193-213, 1989.

[19] H. Ren, L. Chen, C. Shan et al., "Association between sleep disorders and dawn phenomenon in patients with type 2 diabetes mellitus," Zhonghua Yi Xue Za Zhi, vol. 95, no. 16, pp. 1209-1213, 2015.

[20] F. Porcellati, P. Lucidi, G. B. Bolli, and C. G. Fanelli, "Thirty years of research on the dawn phenomenon: lessons to optimize blood glucose control in diabetes," Diabetes Care, vol. 36, no. 12, pp. 3860-3862, 2013.

[21] M. F. Bouchonville, J. J. Jaghab, E. Duran-Valdez, R. M. Schrader, and D. S. Schade, "The effectiveness and risks of programming an insulin pump to counteract the dawn phenomenon in type 1 diabetes," Endocrine Practice, vol. 20, no. 12, pp. 1290-1296, 2014.

[22] H. Yagasaki, K. Kobayashi, T. Saitou et al., "Nocturnal blood glucose and IGFBP-1 changes in type 1 diabetes: differences in the dawn phenomenon between insulin regimens," Experimental and Clinical Endocrinology \& Diabetes, vol. 118, no. 3, pp. 195-199, 2010.

[23] S. Reutrakul and E. Van Cauter, "Interactions between sleep, circadian function, and glucose metabolism: implications for risk and severity of diabetes," Annals of the New York Academy of Sciences, vol. 1311, no. 1, pp. 151-173, 2014.

[24] Z. H. Guo, J. Xu, J. Y. Wang et al., "Correlation study between obesity and dawn phenomenon in patients with type 2 diabetes," Zhonghua Nei Ke Za Zhi, vol. 55, no. 1, pp. 16-20, 2016.

[25] J. Qian, B. Yeh, K. Rakshit, C. S. Colwell, and A. V. Matveyenko, "Circadian disruption and diet-induced obesity synergize to promote development of beta-cell failure and diabetes in male rats," Endocrinology, vol. 156, no. 12, pp. 4426-4436, 2015.

[26] A. B. Hansen, L. Stayner, J. Hansen, and Z. J. Andersen, "Night shift work and incidence of diabetes in the Danish nurse cohort," Occupational and Environmental Medicine, vol. 73, no. 4 , pp. 262-268, 2016.
[27] V. G. Vimalananda, J. R. Palmer, H. Gerlovin et al., "Nightshift work and incident diabetes among African-American women," Diabetologia, vol. 58, no. 4, pp. 699-706, 2015.

[28] A. Bannai, E. Yoshioka, Y. Saijo, S. Sasaki, R. Kishi, and A. Tamakoshi, "The risk of developing diabetes in association with long working hours differs by shift work schedules," Journal of Epidemiology, vol. 26, no. 9, pp. 481-487, 2016.

[29] S. M. Reppert and D. R. Weaver, "Molecular analysis of mammalian circadian rhythms," Annual Review of Physiology, vol. 63, no. 1, pp. 647-676, 2001.

[30] R. D. Rudic, P. McNamara, A. M. Curtis et al., "BMAL1 and CLOCK, two essential components of the circadian clock, are involved in glucose homeostasis," PLoS Biology, vol. 2, no. 11, p. e377, 2004.

[31] Y. Zhao, Y. Zhang, M. Zhou, S. Wang, Z. Hua, and J. Zhang, "Loss of mPer2 increases plasma insulin levels by enhanced glucose-stimulated insulin secretion and impaired insulin clearance in mice," FEBS Letters, vol. 586, no. 9, pp. 1306$1311,2012$.

[32] K. A. Lamia, S. J. Papp, R. T. Yu et al., "Cryptochromes mediate rhythmic repression of the glucocorticoid receptor," Nature, vol. 480, no. 7378, pp. 552-556, 2011. 


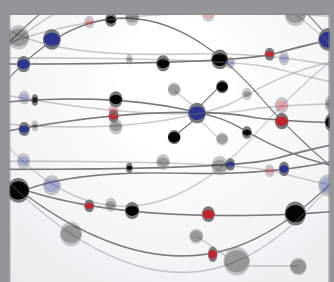

The Scientific World Journal
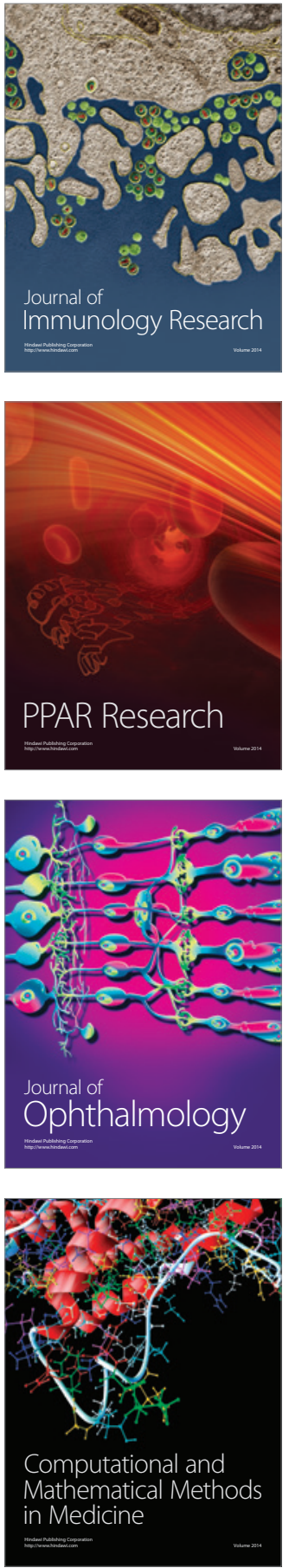

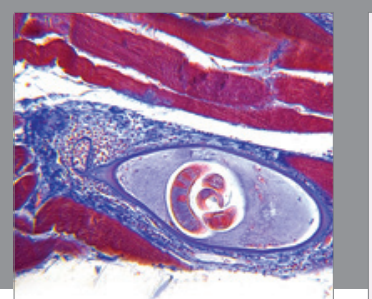

Gastroenterology Research and Practice
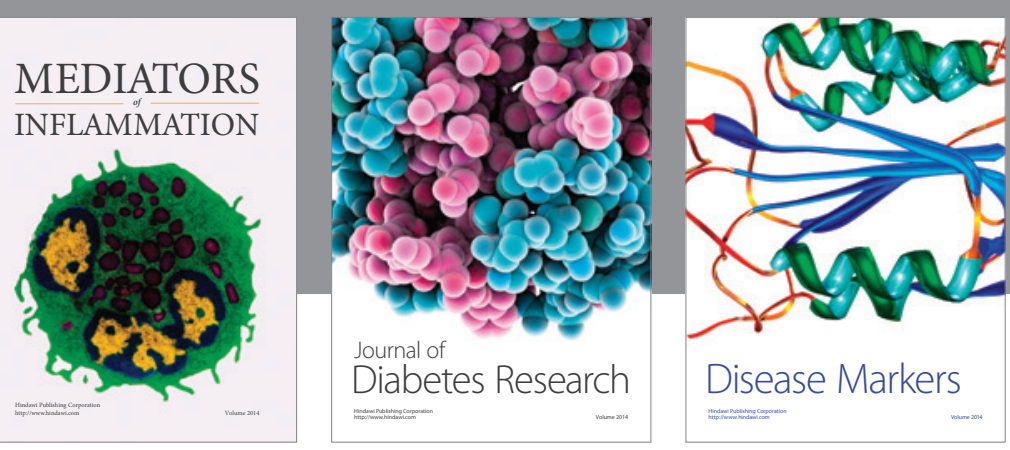

Disease Markers

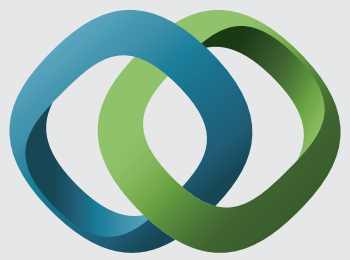

\section{Hindawi}

Submit your manuscripts at

https://www.hindawi.com
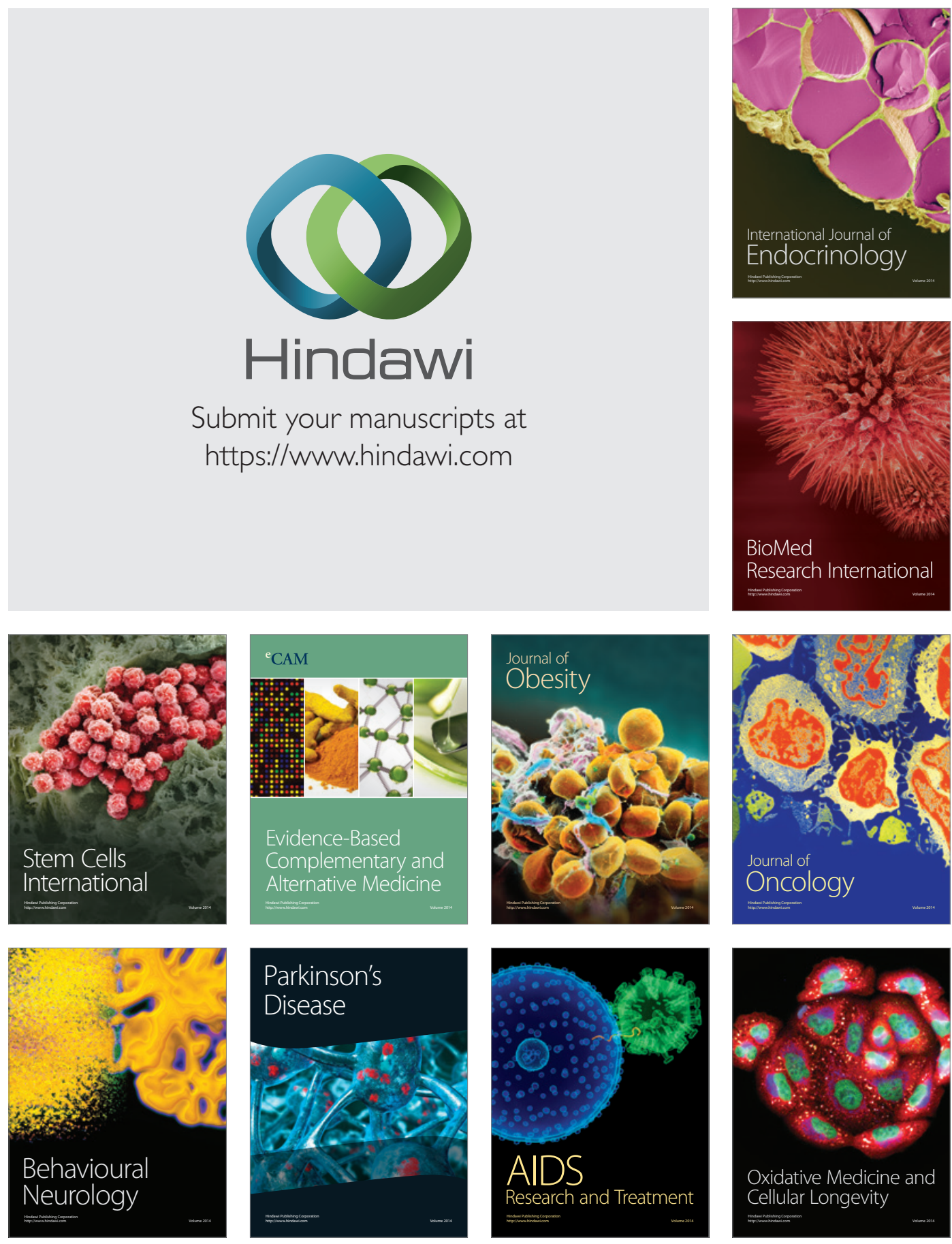